\title{
Non-linear Static Analysis of Offshore Steep Wave Riser
}

\author{
Hongdong Qiao ${ }^{1, a}$, Weidong Ruan ${ }^{1}$, Zhaohui Shang ${ }^{1}$, Yong Bai ${ }^{1}$ \\ ${ }^{1}$ College of Civil Engineering and Architecture, Zhejiang University, Hangzhou, China
}

\begin{abstract}
A new solution combining finite difference method and shooting method is developed to analyze the behavior of steep wave riser suffering from current loading. Based on the large deformation beam theory and mechanics equilibrium principle, a set of non-linear ordinary differential equations describing the motion of the steep wave riser are obtained. Then, finite difference method and shooting method are adopted and combined to solve the ordinary differential equations with zero moment boundary conditions at both the seabed end and surface end of the steep wave riser. The resulting non-linear finite difference formulations can be solved effectively by NewtonRaphson method. To improve iterative efficiency, shooting method is also employed to obtain the initial value for Newton-Raphson method. Results are compared with that of FEM by OrcaFlex, to verify the accuracy and reliability of the numerical method.
\end{abstract}

\section{Introduction}

To overcome harsh environments and high pressure, six main configurations of steel catenary riser have been developed, such as free hanging catenary, lazy wave, steep wave, lazy S, steep S and pliant wave. Each configuration has its advantage to make itself better appropriate for particular applications. Among these configurations, steep wave riser is considered better to accommodate the geometry in deep water, and it is suitable for applications where the riser terminates at a subsea base or completion. As shown in Figure 1, typical steep wave riser is composed of a taut lower pipe connected to a positively buoyant section of catenary which itself joins the sagging part of the riser which in turn ends at the vessel [1]. It is similar to the lazy wave configuration, but has a nearly vertical connection at the seabed where a subsea base controls the touch-down point. Steep wave riser could accommodate large vessel offsets and effectively reduce the potential compression buckling at TDP. However, it requires a subsea base and subsea bend stiffener, and yet is able to maintain its configuration even if the riser fluid density changes.

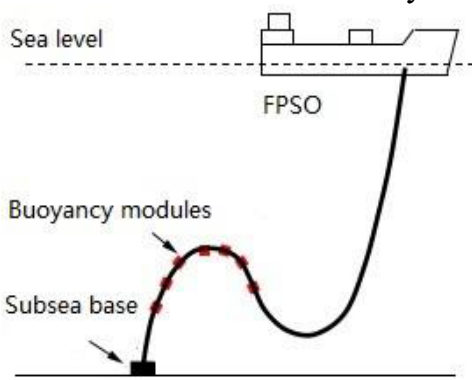

Figure 1. Configuration of steep wave riser

Research on steep wave riser is relatively few. Pinto and Lima [2] presented the final results of FSO steepwave riser configuration design in the deepwater $(815 \mathrm{~m})$ Roncador field and gave a complete report of that project. Then, numerical simulations by Riflex were performed to study the influence of the armour on the response of the steep-wave riser in $300 \mathrm{~m}$ water depth under irregular waves [3]. Sun and Bo [4] adopted the lumped mass method to perform the global analysis on the steep wave flexible riser, and found that the steep wave riser is not very sensitive to the ocean current. Steep wave configuration and lazy wave configuration used on the Auger TLP were specifically developed for application with a tanker FPSO located in a harsh environment [5]. And key mechanical design issues and material selection for different service conditions are discussed and budgetary costs for a range of typical field application were also presented in this paper. Santillan S.T. [6] put forward an elastic approach to describe the analysis of steep-wave flexible risers by a finite difference technique, and a free-vibration analysis was also conducted for small-amplitude oscillations.

This paper presents a numerical method to study the static two-dimensional behavior of steep wave riser by taking the effect of current loading into account. Due to the lifting effect of buoyancy modules attached to part of the riser, the behavior of the steep wave riser is subjected to large deformation and behaves great nonlinearity. Therefore, based on the large deformation beam theory and mechanics equilibrium principle, a set of non-linear ordinary differential equations describing the motion of the steep wave riser are developed. Then, finite difference method and shooting method are combined effectively to solve the ordinary differential equations with zero moment boundary conditions at both the seabed end and surface end of the steep wave riser. The resulting nonlinear finite difference formulations can be solved by the Newton-Raphson method with the Jacobian matrix evaluated in an approximate manner. To verify the accuracy of the proposed method, FEM by OrcaFlex is employed and the results from the two methods show excellent consistency.

\section{Configuration calculation}

The behavior of the steep wave riser is described in terms of the position of the centerline of the riser according to the classical theory of rods [7]. Due to the attachment of buoyancy modules, the configuration of 
steep wave riser can be divided into three segments, as shown in Figure 2:

(1)Touch-down segment, it is the riser from the touchdown point (TDP) to the decline point (DP);

(2)Buoyancy segment, it is the riser from the decline point (DP) to the lift point (LP);

(3)Hang-off segment, it is the riser from the lift point (LP) to the hang-off point (HOP) at the vessel or platform.

In this figure, one global coordinate system ( $\mathrm{x}, \mathrm{y})$ is established in the steep wave riser model by locating the origin at the touch-down point (TDP). And ocean current is set to be positive when flowing in the positive $\mathrm{x}$ direction.

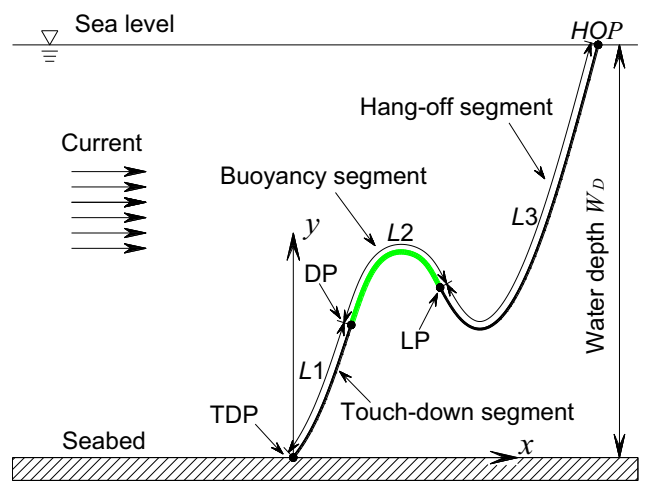

Figure 2. Steep wave riser configuration

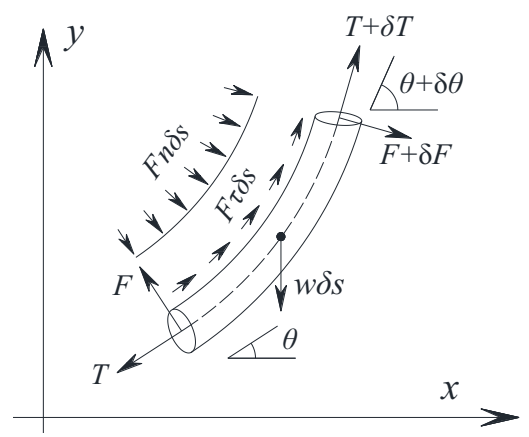

Figure 3. Large-angle deflections-forces acting on the touch-down segment

\subsection{Touch-down segment}

The arc length of the touch-down segment is L1. It is connected vertically to the seabed foundation by a subsea base, at the touch-down point. Due to the lifting force by the buoyancy segment and the displacement constraint of the subsea base, the configuration of the touch-down segment behaves like part of a simple catenary riser. Thus it can be seen that the behavior of the touch-down segment is subject to large-angle deflections. Based on the large deformation beam theory, the basic differential equations for the touch-down segment can be obtained from the force system acting on a differential segment of the touch-down segment, as illustrated in Figure 3 [8].

Resolving forces normal to the differential segment axis leads to

$(F+\delta F) \cos \delta \theta-(T+\delta T) \sin \delta \theta-F+w \delta s \cos \theta+F_{n} \delta s=0$

$$
F_{n}=\frac{1}{2} \rho_{w} C_{d} D d s\left|v_{c} \sin \theta\right| v_{c} \sin \theta
$$

Where $\mathrm{F}$ and $\mathrm{T}$ represent shear force and axial tension, respectively; $\delta \mathrm{F}$ and $\delta \mathrm{T}$ represent the increment of shear force and axial tension; $\theta$ is the inclination angle of the differential segment from horizontal direction to axial direction; $\delta \theta$ represents the increment of the inclination angle; $w$ is the submerged weight per unit length of the riser; $\delta s$ is the length of the differential segment; $\rho w$ is sea density; vc is current velocity; $\mathrm{D}$ is the outer diameter of the riser; Fn represents the drag force in the normal direction per unit length of the riser, acting perpendicular to the riser axis. The drag force in the normal direction Fn can be obtained by Morison's equation.

As the length of the differential segment $\delta$ s is very small, the increment variable $\delta \theta$ could be infinitely small. Therefore, $\cos (\delta \theta) \approx 1$ and $\sin (\delta \theta) \approx \delta \theta$. For simplification, Equation (1) then can lead to

$$
\frac{d F}{d s}-T \frac{d \theta}{d s}+w \cos \theta+F_{n}=0
$$

Furthermore, the shear force $\mathrm{F}$ herein is related to the bending moment by $\mathrm{F}=\mathrm{dM} / \mathrm{ds}$. Hence, Equation (3) can be derived as following:

$$
\frac{d^{2} M}{d s^{2}}-T \frac{d \theta}{d s}+w \cos \theta+F_{n}=0
$$

Based on the large deflection beam theory, the bending moment then can be obtained:

$$
M=E I \kappa=E I \frac{d \theta}{d s}
$$

Where, EI denotes the flexural stiffness of the riser and $\kappa$ denotes the curvature of the riser.

Combining Equation (4) and Equation (5), the final normal mechanics equilibrium differential equation can be expressed as:

$$
E I \frac{d^{3} \theta}{d s^{3}}-T \frac{d \theta}{d s}+w \cos \theta+F_{n}=0
$$

On the other hand, resolving forces parallel to the segment axis leads to

$$
\begin{aligned}
& (F+\delta F) \sin \delta \theta+(T+\delta T) \cos \delta \theta-T-w \delta s \sin \theta+F_{\tau} \delta s=0 \\
& F_{\tau}=\frac{1}{2} \rho_{w} C_{\tau} \pi D d s\left|v_{c} \cos \theta\right| v_{c} \cos \theta
\end{aligned}
$$

Where, F $\tau$ represents the drag force in the axial direction per unit length of the riser, and $\mathrm{C} \tau$ is the drag coefficient in the axial direction.

For simplification, Equation (7) then can be simplified as:

$$
F \frac{d \theta}{d s}+\frac{d T}{d s}-w \sin \theta+F_{\tau}=0
$$

Then, the final axial mechanics equilibrium differential equation can be expressed as: 


$$
E I \frac{d^{2} \theta}{d s^{2}} \frac{d \theta}{d s}+\frac{d T}{d s}-w \sin \theta+F_{\tau}=0
$$

As both the axial deformation and shear deformation are not considered in the analysis, the horizontal and vertical displacements in the global coordinate $(x, y)$ then can be expressed:

$$
x=\int \cos \theta d s, y=\int \sin \theta d s
$$

Once the displacements, inclination angle and curvature along the riser are obtained, the bending moment $\mathrm{M}$ and shear force $\mathrm{F}$ can be derived as follows:

$$
\begin{aligned}
& M=E I \kappa=E I \frac{d \theta}{d s} \\
& F=\frac{d M}{d s}=E I \frac{d^{2} \theta}{d s^{2}}
\end{aligned}
$$

It should be noted that shear force is set to be positive when in clockwise direction.

\subsection{Buoyancy segment}

The arc length of the buoyancy segment is L2. To decrease the upper end tension required at the vessel and decouple the vessel motions from the touch-down point of the riser, buoyancy modules made of syntactic foam or thin-walled air can are uniformly attached along the long length of the buoyancy segment. As the buoyancy force of the buoyancy modules and riser in the buoyancy segment is larger than their weights, the configuration behaves like an arch bend. To simplify the numerical analysis, a uniformly distributed lifting force is acting on the buoyancy segment, which is equivalent to the loading combination of the buoyancy force and weight. The uniformly distributed lifting force here is called "equivalent submerged weight unit length". Besides, the equivalent outer diameter and equivalent flexural stiffness are considered in the buoyancy segment. The adoption of the equivalent outer diameter here is to make sure that the buoyancy force of the equivalent buoyancy segment is equal to the buoyancy combination of the buoyancy modules and riser unit length. And the equivalent flexural stiffness is considered, due to some effect of these buoyancy modules on the flexural stiffness of the buoyancy segment. However, the equivalent flexural stiffness of the buoyancy segment is generally close to the flexural stiffness of the riser due to the small length of buoyancy modules attached. Thus it is generally acknowledged that the equivalent flexural stiffness of the buoyancy segment is equal to the flexural stiffness of the riser. The vertical displacement of the highest point in the arch bend is herein set as yarch. Due to its largedeflection behavior, the configuration analysis of the buoyancy segment is also based on the large deflection beam theory. According to the mechanics equilibrium principle, the governing differential equations for the buoyancy segment are similar with those of the touchdown segment. The main difference is that the flexural stiffness EI, submerged weight $\mathrm{w}$ and outer diameter D in
Equations (1)-(13) should be replaced by the equivalent values EIe, we and De, respectively.

\subsection{Hang-off segment}

The arc length of the hang-off segment is L3. It is hung off by a ball joint to prevent high bending moment induced by wave, current and vessel motion, at the hangoff location. However, as the study is confined to the two-dimensional static analysis, the effects of wave and vessel motions are not considered in this study. Due to the lifting effect of the buoyancy segment and the vessel, the configuration of the hang-off segment behaves like a sag bend. The vertical displacement of the lowest point in the sag bend herein is set as ysag. As the mechanical behavior of the hang-off segment is precisely the same with that of the touch-down segment, the governing differential equations for the hang-off segment are also the same with that of the touch-down segment.

\subsection{Boundary conditions}

The touch-down location of the steep wave riser is generally connected to a subsea base, controlling the touch-down point. Therefore, the boundary condition of the touch-down point in the global coordinate system ( $\mathrm{x}$, y) can be expressed as:

$$
M(0)=0
$$

On the other hand, the hang-off location of the steep wave riser is usually hinged to the vessel or platform by a ball joint. And the inclination angle of the hang-off point can be measured by instrument. Thus, the boundary condition of the hang-off point in the global coordinate system $(\mathrm{x}, \mathrm{y})$ can be expressed as:

$$
\begin{aligned}
& y\left(L_{1}+L_{2}+L_{3}\right)=W_{D} \\
& \theta\left(L_{1}+L_{2}+L_{3}\right)=\theta \\
& M\left(L_{1}+L_{2}+L_{3}\right)=0
\end{aligned}
$$

Where, WD represents water depth.

\section{Numerical solution}

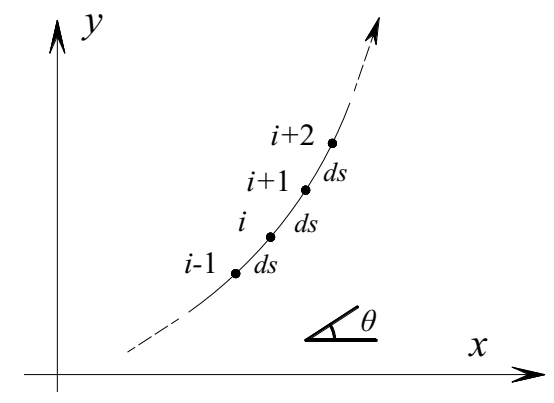

Figure 4. Forces acting on the touch-down segment

In this study, the behavior of the steep wave riser is mainly dominated by Equation (6) and Equation (10). According to the two differential equilibrium equations and boundary conditions, it can be found that the essence of steep wave riser's global analysis is a multipoint boundary value problem. Thus the finite difference 
method is applied to solve the two differential equations numerically in terms of the coordinates of adjacent nodes. For the case of equally spaced nodes, it can be expressed at node $i$, in terms of the element length ds between nodes and the angles at four nodes: the node itself, the two nodes immediately above it, and the one node immediately below it, as shown in Figure 4. The node number here is marked from 1 to $(n+1)$, where the touchdown point is set as node 1 and the hang-off point is set as node $(n+1)$. By considering the effect of ocean current, equations (6) and (10) can be expressed numerically in finite difference form by equations (18) and (19) respectively:

$E I \frac{\theta_{i+2}-3 \theta_{i+1}+3 \theta_{i}-\theta_{i-1}}{(d s)^{3}}-T_{i} \frac{\theta_{i+1}-\theta_{i}}{d s}+w \cos \theta_{i}+\frac{1}{2} \rho_{w} C_{d} D d s\left|v_{c} \sin \theta_{i}\right| \nu_{c} \sin \theta_{i}=0 \quad(i=1 \sim n)$

$$
E I \frac{\theta_{i+1}-2 \theta_{i}+\theta_{i-1}}{(d s)^{2}} \cdot \frac{\theta_{i+1}-\theta_{i}}{d s}+\frac{T_{i+1}-T_{i}}{d s}-w \sin \theta_{i}+\frac{1}{2} \rho_{w} C_{\tau} \pi D d s\left|v_{c} \cos \theta_{i}\right| \nu_{c} \cos \theta_{i}=0 \quad(i=1 \sim n)
$$

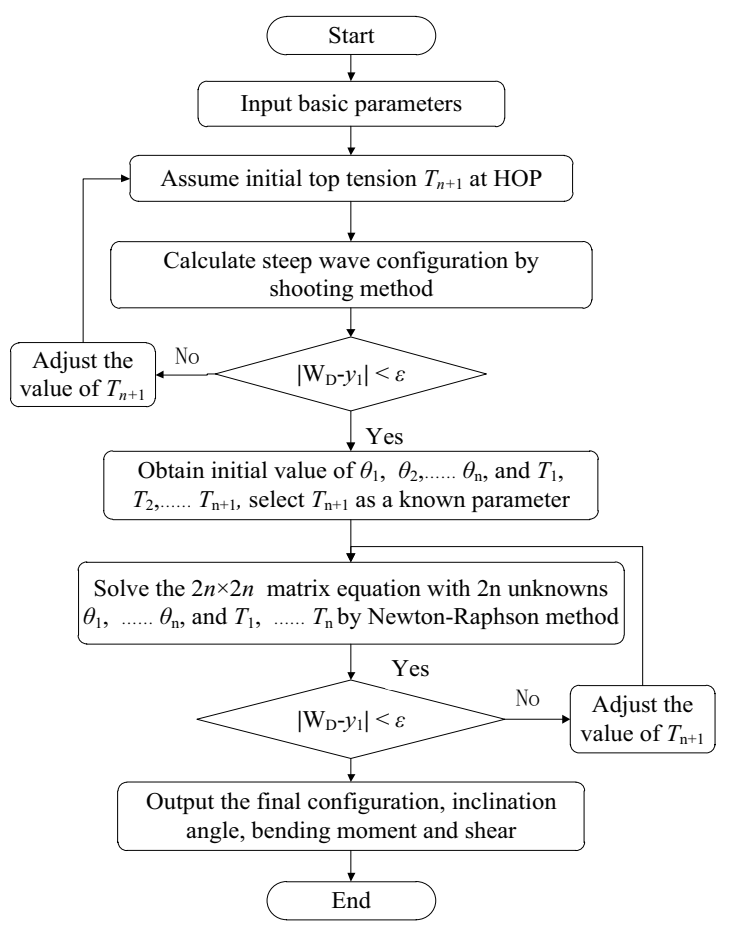

Figure 5. Flow chart of numerical calculation

When the length of the riser reaches between L1 and $(\mathrm{L} 1+\mathrm{L} 2)$, that is to say when calculating the behavior of boundary segment, the flexural stiffness EI, submerged weight $\mathrm{w}$ and outer diameter $\mathrm{D}$ should be replaced by the equivalent values EIe, we and De, respectively. Then a $2 n \times 2 n$ matrix equation can be formulated containing $n+3$ angle unknowns $(\theta 0, \theta 1, \theta 2, \cdots \cdots, \theta n+1$ and $\theta n+2)$ and $\mathrm{n}+1$ tension unknowns (T1, T2, $\cdots, \cdots, \mathrm{Tn}$ and $\mathrm{Tn}+1)$. According to the boundary condition (14) and (17), the value of $\theta n+2$ can be expressed in terms of $\theta n+1$ and $\theta n$, and $\theta 0$ in terms of $\theta 1$ and $\theta 2$. Besides, the value of $\theta n+1$ can be obtained from the boundary condition (16). Finally, it can be found that there are still $2 n+1$ unknowns $(\theta 1, \theta 2, \cdots \cdots, \theta n$ and $\mathrm{T} 1, \mathrm{~T} 2, \cdots \cdots, \mathrm{Tn}+1)$ left in the matrix equation. The above problem can be effectively solved by shooting method. In addition, to improve the efficiency of Newton-Raphson iteration method, the initial assumed parameters used in this iteration method could be derived by shooting method [9]. Figure 5 shows the main procedures of the numerical solution. In the flow chart, $\varepsilon$ respects the specified small quantity.

\section{Comparison and discussion}

In order to verify the accuracy and reliability of the numerical method, comparisons are performed with the results obtained from the proposed method and FEM by OrcaFlex. To perform the analysis, basic properties of the riser and environment are shown in Table 1 and Table 2, respectively. Comparisons of steep wave configuration, tension, bending moment and shear force are shown in Figures 6-9, respectively. From these figures, it can be seen that the results obtained from the two methods coincide well. It can strongly verify the accuracy and reliability of the proposed method. Differences between the numerical method and FEM by OrcaFlex are shown in Table 3

Table 1. Riser parameters

\begin{tabular}{|l|l|}
\hline Items & Value \\
\hline$L_{1}(\mathrm{~m})$ & 450 \\
\hline$L_{2}(\mathrm{~m})$ & 672 \\
\hline$L_{3}(\mathrm{~m})$ & 1076 \\
\hline$w(\mathrm{~N} / \mathrm{m})$ & 501.7 \\
\hline$w_{\mathrm{e}}(\mathrm{N} / \mathrm{m})$ & -785.9 \\
\hline$D(\mathrm{~m})$ & -0.220 \\
\hline$D_{\mathrm{e}}(\mathrm{m})$ & -0.460 \\
\hline$E I\left(\mathrm{KN} \cdot \mathrm{m}^{2}\right)$ & $4.736 \mathrm{E} 2$ \\
\hline
\end{tabular}

(Note: the flexural stiffness of the boundary segment is assumed to be the same with the other two segments, that is to say $\mathrm{EI}=\mathrm{EIe}$.)

Table 2. Environmental parameters

\begin{tabular}{|c|c|}
\hline Items & Value \\
\hline$W_{D}(\mathrm{~m})$ & 1500 \\
\hline$\rho\left(\mathrm{kg} / \mathrm{m}^{3}\right)$ & 1025 \\
\hline$v_{c}(\mathrm{~m} / \mathrm{s})$ & 0.2 \\
\hline$\theta(\mathrm{deg})$ & 80 \\
\hline$C_{d}$ & 0.7 \\
\hline$C_{\tau}$ & 0.008 \\
\hline
\end{tabular}


Table 3. Results of numerical method VS FEM by OrcaFlex

\begin{tabular}{|c|c|c|c|}
\hline Items & $\begin{array}{c}\text { Numerical } \\
\text { method }\end{array}$ & $\begin{array}{c}\text { FEM by } \\
\text { OrcaFlex }\end{array}$ & $\begin{array}{c}\text { Difference } \\
(\%)\end{array}$ \\
\hline$x_{H O P}(\mathrm{~m})$ & 1018 & 1012 & 0.59 \\
\hline$y_{\text {arch }}(\mathrm{m})$ & 891.6 & 893.2 & 1.79 \\
\hline$y_{\text {sag }}(\mathrm{m})$ & 755.1 & 755.1 & 0.01 \\
\hline $\begin{array}{c}T_{H O P} \\
(\mathrm{KN})\end{array}$ & 454.7 & 454.7 & 0.00 \\
\hline $\begin{array}{c}T_{T D P} \\
(\mathrm{KN})\end{array}$ & 225.9 & 226.4 & 0.22 \\
\hline $\begin{array}{c}M_{\text {arch }} \\
(\mathrm{KN} \cdot \mathrm{m})\end{array}$ & -4.520 & -4.588 & 1.48 \\
\hline $\begin{array}{c}M_{\text {sag }} \\
(\mathrm{KN} \cdot \mathrm{m})\end{array}$ & 2.919 & 2.939 & 0.68 \\
\hline $\begin{array}{c}F_{D P} \\
(\mathrm{KN})\end{array}$ & -0.1145 & -0.1187 & 3.54 \\
\hline $\begin{array}{c}F_{L P} \\
(\mathrm{KN})\end{array}$ & 0.8261 & 0.8258 & 0.04 \\
\hline
\end{tabular}

As shown in Figure 6, the steep wave configurations from the two methods seem in perfect consistency. It can be seen that the difference mainly happens at the hang-off segment. The horizontal distances from the hang-off point to the touch-down point obtained from numerical method and FEM by OrcaFlex are $1018 \mathrm{~m}$ and $1012 \mathrm{~m}$ respectively, and the difference is less than $3.0 \%$. Besides, the highest vertical locations of the arch bend from the two methods are $891.6 \mathrm{~m}$ and $893.2 \mathrm{~m}$, respectively. And the lowest vertical locations of the sag bend from the two methods are $755.1 \mathrm{~m}$ and $755.1 \mathrm{~m}$, respectively.

The tensions illustrated in Figure 7 seem also in excellent agreement. It can be found that three local maximum tensions occur at the hang-off point, lift point and decline point, respectively. Among the three local maximum tensions, the tension at the hang-off point seems to be the maximum, arriving at $454.70 \mathrm{KN}$. While under the same condition, the upper end tension in simple free hanging configuration without the addition of buoyancy modules can reach up to $915.63 \mathrm{KN}$, which is greater than that in the steep wave configuration. It can be seen that steep wave configuration can reduce the upper end tension effectively.

Figure 8 shows the comparison of riser's bending moments, and the overall bending moments almost perfectly match. As expected, two local maximum bending moments occur at the peak point in the arch bend and the lowest point in the sag bend. In addition, the values of bending moments at the LP and DP turn out to be zero. And it can be found that the bending moments in the neighborhood of the LP and DP change very obviously. The maximum bending moments in the steep wave configuration from numerical method and FEM by OrcaFlex are $-4.520 \mathrm{KN} \cdot \mathrm{m}$ and $-4.588 \mathrm{KN} \cdot \mathrm{m}$ respectively, which are very close.

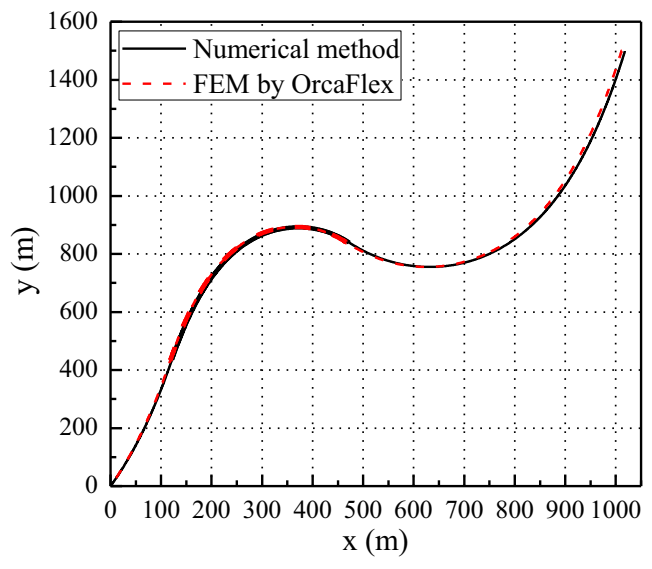

Figure 6. Comparison of steep-wave configurations

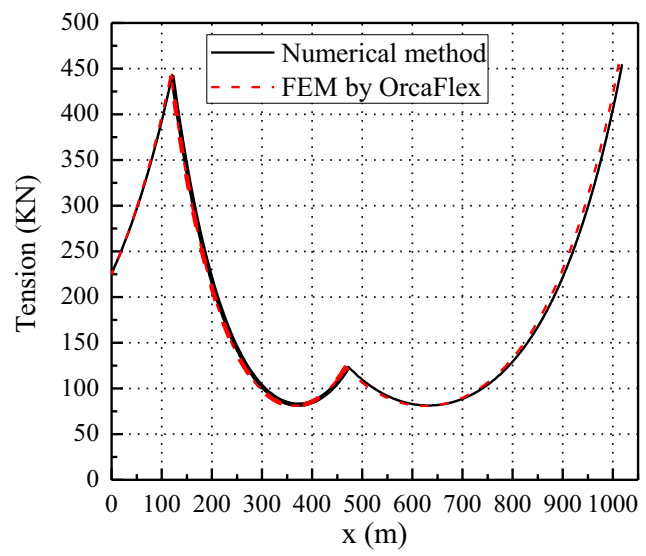

Figure 7. Comparison of steep-wave tensions

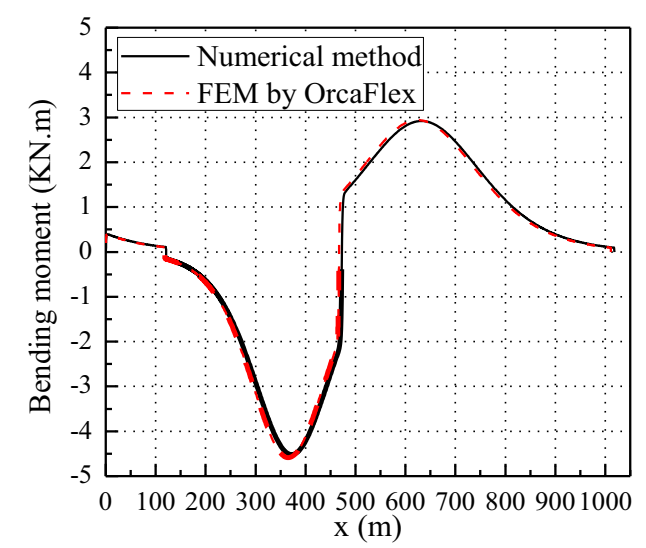

Figure 8. Comparison of steep-wave bending moments

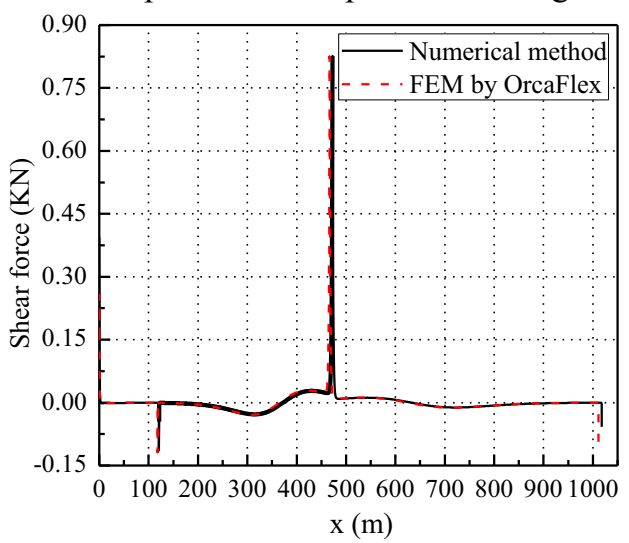

Figure 9. Comparison of steep-wave shear forces 
The comparison of riser's shear forces is shown in Figure 9. The overall shear forces in Figure 9 match very well, except at the two locations of HOP and TDP. Furthermore, two local maximum shear forces occur at the DP and LP and the shear force in other location is very close to zero. The local maximum shear forces in the neighborhood of DP from numerical method and FEM by OrcaFlex are $-0.1145 \mathrm{KN}$ and $-0.1187 \mathrm{KN}$ respectively. And the local maximum shear forces in the neighborhood of DP from numerical method and FEM by OrcaFlex are $0.8261 \mathrm{KN}$ and $0.8258 \mathrm{KN}$ respectively.

\section{Conclusions}

This study presents a reasonable numerical method to study the mechanical behavior of planar steep wave

\section{References}

1. F.B. Seyed, M.H.Patel. Mari. Stru. 5(2), 121(1992)

2. P. Pinto, G.Lima. 20th Int. Conf. on Offs. Mech. and Arct. Engi. 133(2001).

3. B. Bonnemaire, 14th Inte. Offs. and Pola Engi. Conf. 236(2004). riser considering the effect of ocean current. According to mechanics equilibrium principle, two non-linear ordinary differential equations are set to for each differentiation element of steep wave riser. Finite difference method and shooting method are combined to solve the ordinary differential equations and the resulting non-linear finite difference formulations can be solved by the NewtonRaphson method. The accuracy and reliability of the proposed method has been well verified by comparing FEM by OrcaFlex. In conclusion, the comparison analyses have demonstrated the ability of the proposed method in predicting the static behavior of steep wave riser. Further research needs to be carried out on dynamic response of steep wave riser.

4. L. Sun, B. Qi, Mari. Sci. and Appl. 10(4): 478(2011)

5. S. A. Hatton. FPSO Worl. Congr. \& Tech. Exhi, 800(1995)

6. S.T. Santillan, L.N. Virgin, R.H Plaut R.H, J Offs. Mech Arct. 132(2010)

7. D.L. Garrett.. J. Ener. Reso. Tech, 104(1982)

8. C. P.Sparks. Fund. of mari. Rise. Mech: basi. Prin. and simp.anal, (2007).

9. Y.Bai, B.Yu, P.Cheng, The $22^{\text {nd }}$ Intl.Offs.and Pola. Engin. Conf, 2 103(2012) 